\title{
Predictors of Surgical Results in Patients with Primary Pontine Hemorrhage
}

\author{
Chuanyuan $\mathrm{TAO}^{1}$, Hao $\mathrm{LI}^{1}$, Jiajing WANG${ }^{2}$, Chao $\mathrm{YOU}^{1}$ \\ ${ }^{1}$ Sichuan University, West China Hospital, Department of Neurosurgery, Chengdu, China \\ 2Sichuan University, West China Hospital, Department of Neurosurgical ICU, Chengdu, China
}

\section{ABSTRACT}

\begin{abstract}
AIM: Prediction of outcome for surgical patients with primary pontine hemorrhage (PPH) is seldom reported although many predictors from clinical and radiological features have been identified in conservative patients. The purpose of this study was to assess the prognostic factors affecting the 30-day mortality and 3-month functional outcome in surgical patients after PPH.

MATERIAL and METHODS: Forty-five patients with large PPH ( $>5 \mathrm{ml}$ ) and Glasgow Coma Scale (GCS) score $<8$ were treated surgically at West China Hospital. The demographic, clinical, imaging and follow-up data were collected retrospectively. Factors affecting the mortality and functional outcome were statistically analyzed.

RESULTS: Fourteen patients (31.1\%) died within 30 days and 7 patients (15.6\%) gained a favorable functional recovery within 3 months. A multivariate analysis showed that the hematoma volume, GCS score on admission, age, and type of hemorrhage were all significantly related to the 30-day mortality, while the hematoma volume, GCS score on admission, rostrocaudal extension were associated with the 3-month functional outcome. The presence of hydrocephalus was not found responsible for the surgical outcomes.

CONCLUSION: The identification of these prognostic factors is helpful for selecting the candidates for surgical treatment. Those with younger age, smaller hematoma without rostrocaudal extension, unilateral hemorrhage, and higher GCS score may benefit from surgery.
\end{abstract}

KEYWORDS: Primary pontine hemorrhage, Prognosis, Surgery, Mortality, Functional outcome

\section{INTRODUCTION}

Primary pontine hemorrhage (PPH), the most common type of primary/hypertensive brainstem hemorrhage, constitutes approximately $5-10 \%$ of all spontaneous intracranial hemorrhage $(3,11)$. The lesion has a diversified prognosis from recovery without neurological deficit to early death depending on the clinical and radiological conditions $(2,13)$. The overall mortality was reported to be $40-60 \%(6,15)$. Many studies have identified prognostic indicators from the clinical presentations and radiological findings to predict the outcome in $\mathrm{PPH}$ patients. However, the subjects in these studies were mainly medically-treated patients $(1,3,10,15,16)$.
Although the role of surgery in the management of PPH is controversial, several case-control investigations demonstrated that operation could decrease mortality, or even improve functional outcome $(4,6,7,12,14)$. In order to select candidates who would benefit most from surgical procedures, it is essential to decide which factors impacting the surgical outcome.

The aim of this study was to analyze the prognostic factors affecting the 30-day mortality and 3-month favorable functional outcome in $45 \mathrm{PPH}$ patients undergoing hematoma evacuation in acute stage with $\mathrm{PPH}>5 \mathrm{ml}$, Glasgow Coma Scale (GCS) score $<8$ on admission. 


\section{MATERIAL and METHODS}

\section{Patients}

Between January 2003 and September 2013, 45 surgical patients from 110 patients with PPH admitted to our institution were included in this research. Patients were excluded when they had head trauma, coagulopathy and atypical computed tomography (CT) findings suspected of cavernous malformation, aneurysm, arteriovenous malformation (AVM) and tumor apoplexy. We further excluded hemorrhage with a causative lesion such as cavernoma and AVM documented on surgical records. Extremely poor patients with bilaterally dilated pupils, no spontaneous breath, or GCS score 3 on admission were not included. This study has been approved by Ethic Committee of West China Hospital and all patients' guardians have given their informed consent.

\section{Clinical Data}

All patients' clinical data were retrospectively reviewed including age, sex, hypertension (defined as pre-documented systolic blood pressure (BP) $>140 \mathrm{mmHg}$ or diastolic BP $>90 \mathrm{mmHg}$ or the use of anti-hypertensive agent), level of consciousness right after admission, radiological findings, treatment modalities (hematoma evacuation only or hematoma evacuation and external ventricular drainage) and outcome. The level of consciousness was quantified by using the GCS score. The location and volume of hematoma were evaluated on head CT. The intraventricular and rostrocaudal extension and the presence of hydrocephalus were also noted. We divided PPH into three types proposed by Chung and Park (2): massive, basal or bilateral tegmental and unilateral tegmental group. Hemorrhage volume was calculated by the formula $A B C / 2$ where $A$ and $B$ are the perpendicular maximal diameters of the lesion and $C$ is the total length in the vertical plane (8).

\section{Surgical Outcome}

Surgical treatment involved direct hematoma evacuation via different approaches according to hemorrhage features, and ventricular drainage if hydrocephalus coexisted. Outcome was assessed by two indications: 30-day mortality rate and 3-month functional recovery outcome evaluated using Glasgow outcome scale (GOS) score. Favorable outcome was defined as GOS score $\geq 4$.

\section{Statistical Analysis}

Depending on the distribution of the variables, continuous data are described as the mean \pm standard deviation. A univariate analysis was applied first to identify associations between the outcomes and the potential prognostic factors using Chisquare test or Fisher's exact test for categorical data, unpaired t-test and Mann-Whitney $U$ test for normally and non-normally distributed continuous data, respectively. The associated variables $(p<0.10)$ were then entered into multiple logistic regression model to identify independent predictors of 30day mortality and 3-month favorable function outcome. All the above-mentioned statistical analyses were performed using the Statistical Package for the Social Sciences (SPSS) version 18.0 (SPSS Inc., Chicago, IL, USA). The level of significance was accepted at $p$ value $<0.05$.

\section{RESULTS}

\section{Baseline Data}

Of 45 eligible patients with $\mathrm{PPH}>5 \mathrm{ml}$ and $\mathrm{GCS}<8,15$ patients admitted in the first five years (2003-2008) and 30 patients within the recent five years (2008-2013). There were 26 males and 19 females with an age range of $45-70$ years (mean, $62.4 \pm 6.7$ years). 30 patients had a history of hypertension. On admission, the mean GCS score was $5.9 \pm 0.7$, and the mean hematoma volume was $11.6 \pm 4.1 \mathrm{ml}$. According to the CT classification, there were 7 patients in the massive group, 31 in the basal or bilateral tegmental group and 7 in the unilateral tegmental group. Rostrocaudal extension into midbrain/ medulla was detected in 23 patients and ventricular extension in 25 patients. In addition, obstructive hydrocephalus was observed in 20 patients. The clinical and radiological findings, treatment outcomes were summarized in Table I.

\section{Surgical Techniques}

All patients underwent craniotomy under continuous monitoring of brainstem evoked potentials within 12 hours of the onset. The operative approach was designed depending on the location as well as the proximity of hematoma to the pial surface of brainstem (Figures 1A-F; 2A, B; 3A-D; 4A, B; $5 A-E)$. Generally, the unilateral tegmental type was removed by midline or lateral infratentorial approach, while most of the other two types were approached via midline suboccipital exposure. A subtemporal approach was also practiced when the hematoma extended up-toward midbrain mainly in one side. If accompanied with hydrocephalus, ventricular external drainage in the trigone region was initially performed before craniotomy. After the dura was opened, cerebrospinal fluid was fully released by dissecting cisterns or lowering ventricular canal. The cerebellar hemisphere was retracted gently to enter the fourth ventricle or reveal the lateral side of brainstem. Along the broken point in the floor of fourth ventricle or by a small incision precisely over the dark-brown pial aspect, we aspirated the intraparenchymal blood with great care under microscope. Hemostasis was usually realized by Surgicel $\AA$ and continuous irrigation. Fine bipolar coagulation was reserved when measures above failed.

\section{Outcomes}

The 30 -day mortality was $31.1 \%$ (14 patients). Four patients died within 7 days while the rest died after one week within 30 days after operation. Patients who died were significantly older, had lower GCS score and larger hematoma volume in the univariable analysis. The presence of rostrocaudal and ventricular extension were also significantly associated with 30-day mortality. Additionally, death occurred more commonly in the massive group and less frequently in the unilateral group (Table I). However, only age (adjusted odds ratio [OR] $1.259,95 \%$ confidence interval $[\mathrm{Cl}] 1.032-3.573, \mathrm{p}=0.032$ ), GCS score (OR 0.457, Cl 0.124-0.956, $\mathrm{p}=0.024$ ), hematoma volume (OR 1.584, Cl 1.165-1.928, $\mathrm{p}=0.017$ ) and unilateral tegmental type (OR 0.235, Cl 0.084-0.445, $\mathrm{p}=0.038$ ) were identified as significant in the multivariate stepwise backward logistic analysis (Table II). 
Tao C. et al: Predictors of Surgical Results of PPH

Table I: Clinical Characteristics Related to 30-Day Mortality and 3-Month Favorable Outcome in Surgical Patients with PPH

\begin{tabular}{|c|c|c|c|c|c|}
\hline Characteristic & $\begin{array}{c}\text { Baseline } \\
(n=45)\end{array}$ & $\begin{array}{l}\text { 30-day mortality } \\
\quad(n=14)\end{array}$ & p & $\begin{array}{l}\text { Favorable outcome } \\
\qquad(n=7)\end{array}$ & $\mathbf{p}$ \\
\hline Male & $26(57.8)$ & $9(64.3)$ & 0.553 & $3(42.9)$ & 0.433 \\
\hline Age (years) & $62.4 \pm 6.7$ & $68.0 \pm 6.2$ & $<0.001$ & $60.8 \pm 5.9$ & 0.025 \\
\hline Hypertension & $30(66.7)$ & $10(71.4)$ & 0.649 & $4(57.1)$ & 0.670 \\
\hline GCS score & $5.9 \pm 0.7$ & $5.4 \pm 0.9$ & $<0.001$ & $6.4 \pm 0.5$ & $<0.001$ \\
\hline Hematoma volume (ml) & $11.6 \pm 4.1$ & $15.3 \pm 3.5$ & $<0.001$ & $7.8 \pm 2.3$ & $<0.001$ \\
\hline Type of hemorrhage & & & 0.012 & & 0.015 \\
\hline Massive & $7(15.6)$ & $5(35.7)$ & & $0(0)$ & \\
\hline Basotegmental & $31(68.8)$ & $9(64.3)$ & & $3(42.9)$ & \\
\hline Unilateral tegmental & $7(15.6)$ & $0(2.7)$ & & $4(57.1)$ & \\
\hline Rostrocaudal extension & $23(51.1)$ & $11(78.6)$ & 0.013 & $0(0)$ & 0.004 \\
\hline Ventricular extension & $25(55.6)$ & $11(24.4)$ & 0.037 & $2(28.6)$ & 0.214 \\
\hline Hydrocephalus & $20(44.4)$ & $9(64.3)$ & 0.072 & $0(0)$ & 0.012 \\
\hline Admission time & & & 0.820 & & 0.395 \\
\hline Early five years & $15(33.3)$ & $5(35.7)$ & & $1(14.3)$ & \\
\hline Recent five years & $30(66.7)$ & $9(64.3)$ & & $6(85.7)$ & \\
\hline Surgical modality & & & 0.072 & & 0.243 \\
\hline HE+EVD & $20(44.4)$ & $9(64.3)$ & & $1(14.3)$ & \\
\hline $\mathrm{HE}$ & 25 (55.6) & $5(35.7)$ & & $6(85.7)$ & \\
\hline Surgical approach & & & 0.746 & & 0.278 \\
\hline Midline & $32(71.1)$ & $11(78.6)$ & & $4(57.1)$ & \\
\hline Subtemporal & $8(17.8)$ & $2(14.3)$ & & $1(14.3)$ & \\
\hline Lateral infratentorial & $5(11.1)$ & $1(7.1)$ & & $2(28.6)$ & \\
\hline
\end{tabular}

Data are expressed as $n(\%)$, mean \pm standard deviation as appropriate.

PPH: Primary pontine hemorrhage, GCS: Glasgow coma scale, HE: Hematoma evacuation, EVD: External ventricular drainage.

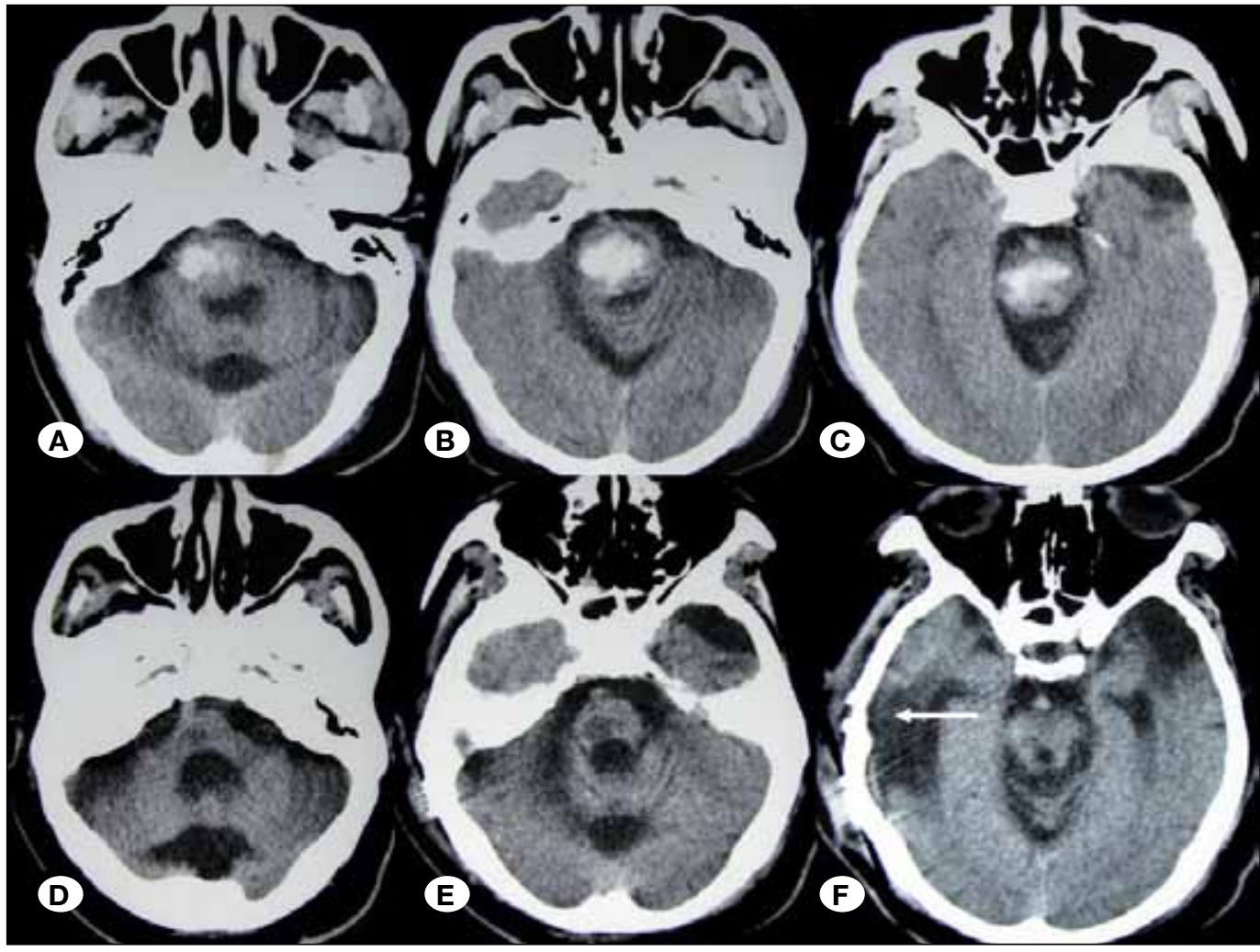

Figure 1: M, $48 \mathrm{y}$, GCS 4 on admission. A-C) Head CT demonstrated massive $\mathrm{PH}$ extending into the midbrain. Most of the hematoma was evacuated urgently via right subtemporal approach 4 hours after onset of first symptom.

D-F) Two months later, postoperative CT showed complete disappearance of hematoma and pontine atrophy. Bone flap and temporal malacia were indicated by arrow. The patient survived in vegetative state (GOS 2). 

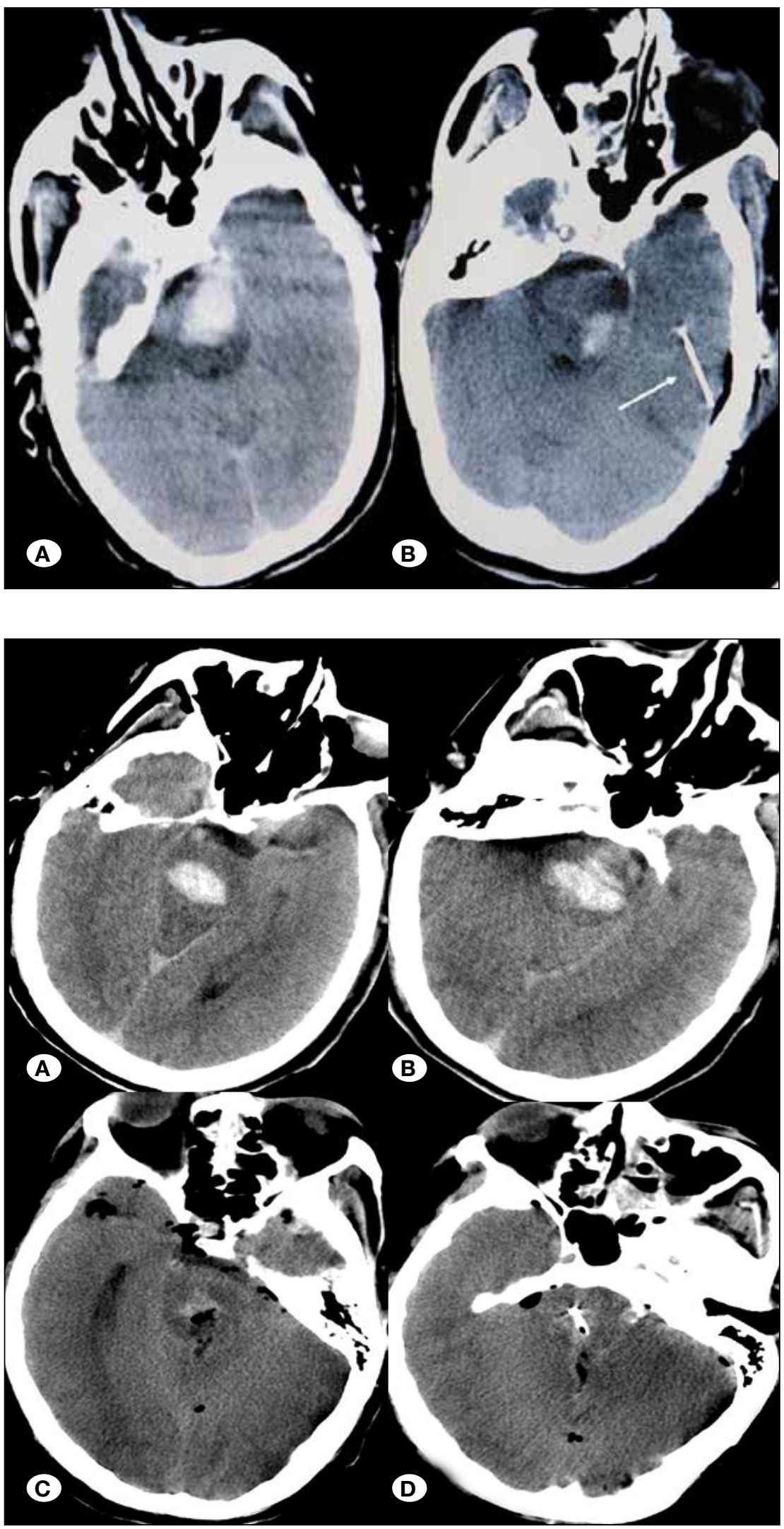

Figure 2: F, 64 y, GCS 6 on admission. A) Preoperative brain $\mathrm{CT}$ revealed $\mathrm{PH}$ of basotegmental type mainly extruding to the left side. She underwent hematoma removal through left subtemporal approach 9 hours after ictus. B) Repeat CT showed a small piece of hematoma left and a drainage tube. She was alert 2 weeks after surgery with 3-month GOS of 4 .
Figure 3: M, 45 y, GCS 5. A, B) Head CT demonstrated bilateral basotegmental hemorrhage of pons. The hematoma was removed via suboccipital midline approach. C, D) Vanish of blood clot was revealed on postoperative CT. He survived with GOS 3. 
The incidence of 3-month favorable functional outcome was $15.6 \%$ (7 patients). Univariable analysis revealed patients with good 3-month outcome were much younger, had significantly higher GCS score, lower hematoma volume. Location of hemorrhage, rostrocaudal extension, and hydrocephalus were also related to favorable functional outcome (Table I). Further multivariable analysis detected GCS score (OR 5.127, $\mathrm{Cl} 1.468-42.834, \mathrm{p}=0.010$ ), hematoma volume (OR 0.239 , $\mathrm{Cl}$ 0.025-0.762, $\mathrm{p}=0.023$ ) and rostrocaudal extension (OR
$0.042, \mathrm{Cl} 0.005-0.182, \mathrm{p}=0.010$ ) as independent factors for 3-month favorable functional outcome (Table II).

\section{DISCUSSION}

Despite PPH usually causes coma, vital sign instability, pupillary abnormality and sensory and motor deficits, they are noted to have a much less uniform prognosis from early death to long-term survival with intact neurological function.

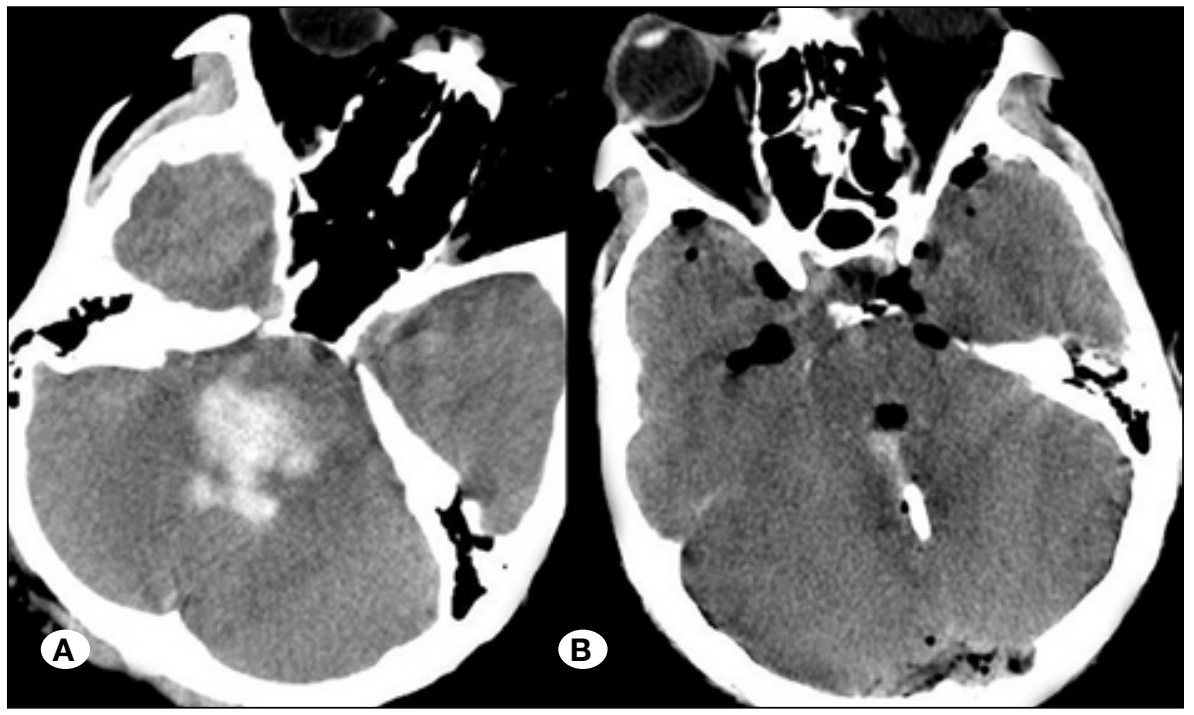

Figure 4: F, 59 y, GCS 7. A) Bilateral tegmental hematoma of pons breaking into the fourth ventricle was clearly seen on initial cranial CT. We removed the hematoma urgently via suboccipital approach. B) The hematoma in ventricle and within the brainstem was totally cleared on CT three days after operation. He was alert with GOS score of 3 .

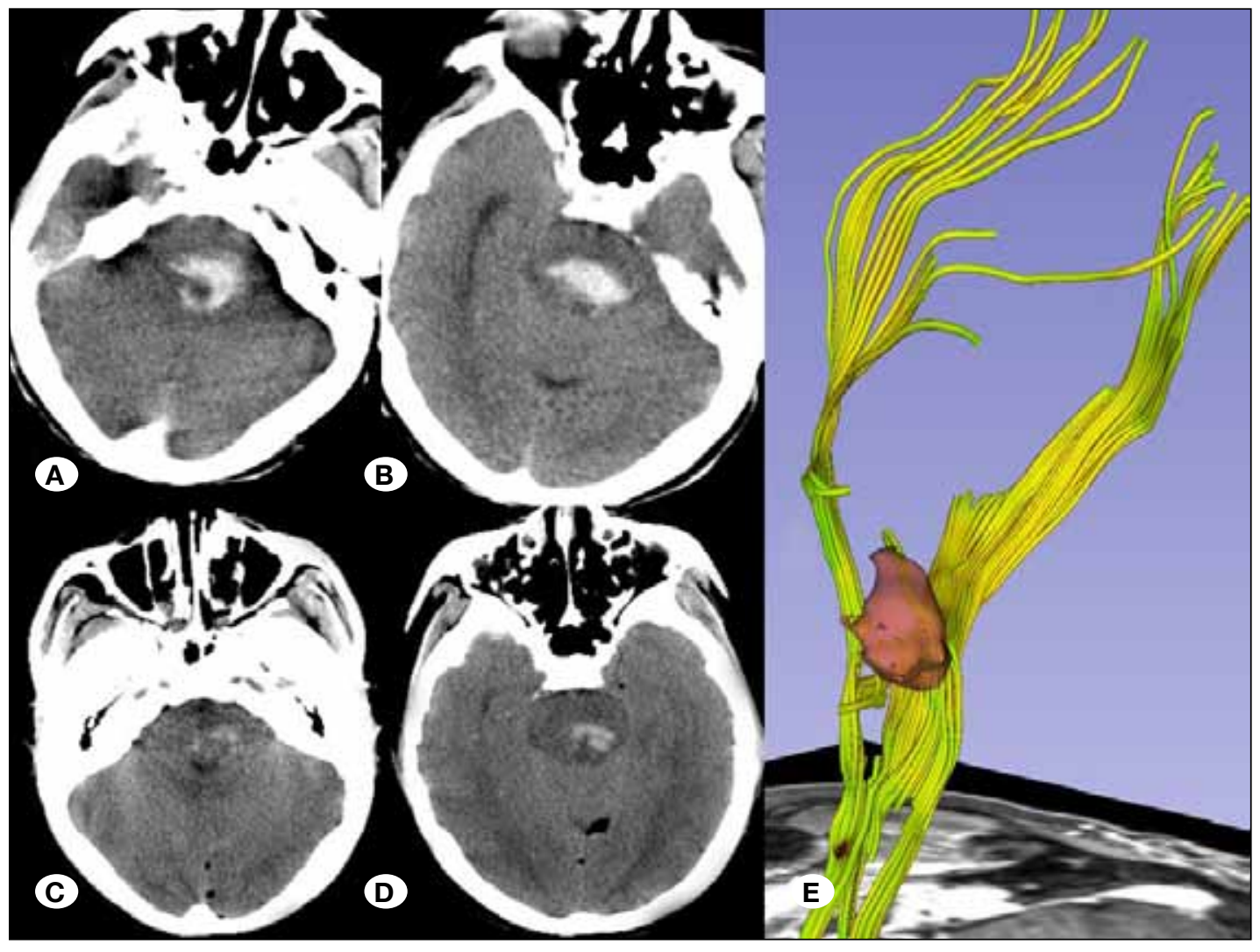

Figure 5: $F, 61 \mathrm{y}, \mathrm{GCS} 7$. A, B) Head CT showed a pontine hematoma of $5 \mathrm{ml}$ mainly limited in the right tegmental area. C, D) Suboccipital craniotomy was done immediately with the majority of blood clot been removed. The consciousness returned to normal soon after surgery.

E) The diffusion tensor imaging (DTI) two weeks later demonstrated that the left pyramidal tract was intact although compressed to be tenuous by residual hematoma posterolaterally. He recovered with GOS 5 . 
Table II: Multiple Logistic Regressions of Factors Related to 30-Day Mortality and 3-Month Favorable Outcome in Surgical Patients After $\mathrm{PPH}$

\begin{tabular}{|c|c|c|c|c|}
\hline \multirow{2}{*}{ Characteristic } & \multicolumn{2}{|c|}{ 30-day mortality } & \multicolumn{2}{|c|}{ Favorable outcome } \\
\hline & OR (Cl) & $P$ value & OR $(\mathrm{Cl})$ & $P$ value \\
\hline Age & $1.259(1.032-3.573)$ & 0.032 & $1.027(0.768-1.459)$ & 0.181 \\
\hline Hematoma volume (ml) & $1.584(1.165-1.928)$ & 0.017 & $0.239(0.025-0.762)$ & 0.023 \\
\hline \multicolumn{5}{|l|}{ Location of hemorrhage } \\
\hline Massive & reference & & reference & \\
\hline Rostrocaudal extension & $4.924(0.842-32.854)$ & 0.145 & $0.042(0.005-0.182)$ & 0.010 \\
\hline Ventricular extension & $2.328(0.365-8.802)$ & 0.855 & excluded & \\
\hline Hydrocephalus & 3.739 (0.262-42.328) & 0.385 & $1.284(0.164-19.843)$ & 0.984 \\
\hline $\begin{array}{l}\text { Surgical modality } \\
\qquad \mathrm{HE}+\mathrm{EVD} \text { VS HE }\end{array}$ & $1.154(0.012-6.935)$ & 0.138 & excluded & \\
\hline
\end{tabular}

PPH: Primary pontine hemorrhage, GCS: Glasgow coma scale, OR: Odds ratio, Cl: Confidence interval, HE: Hematoma evacuation, EVD: External ventricular drainage.

Large hematoma and severe disturbance of consciousness are commonly recognized as good predictors of mortality and unfavorable functional outcome in patients under medical treatment $(3,9,10,15)$. It has been reported that survival is impossible if all 3 features including large paramedian pontine hemorrhage $(\mathrm{PH})$, transverse diameter of hematoma $\geq 20$ $\mathrm{mm}$, and coma on admission are present in $\mathrm{PPH}$ patients (3). However, the influence of clinical or radiological factors on surgical prognosis after PPH was never investigated, especially in critical patients with large hemorrhage and low level of consciousness. This study revealed that surgical outcome was affected not only by hematoma volume and severity of consciousness (both 30-day mortality and 3-month functional outcome), but also by age and type of hemorrhage (30-day mortality), and rostrocaudal extension (3-month functional outcome).

It is well documented that either a large hematoma volume or a lower level of consciousness on admission is associated with higher mortality and worse functional outcome $(6,15,16)$. Shitamichi et al. reported that the patients with a hematoma volume of $5-10 \mathrm{ml}$ had a better outcome than those with a hematoma volume over $10 \mathrm{ml}$ after hematoma aspiration (12). Takahama et al. observed in their study that stereotactic aspiration could produce functional favorable effect in cases of slighter disturbance of consciousness (14). However, in Hong et al.'s series, it seemed that preoperative GCS score failed to correlate with postoperative GOS score in their late craniectomy group (5). Our study suggested that GCS score on admission independent on hematoma volume had an impact on surgical results although a large hematoma usually leads to a severe unconscious state.

Hemorrhagic type was highly predictive of death. Unilateral tegmental hemorrhage was significantly related to survival as all 7 patients in this group survived whereas 5 out of 7 patients in massive group died after surgery. Bilateral basotegmental type had the modest mortality but showed no statistical significance in the final analysis. The possible explanation may be that the primary brain injury to the vital structures within the pons including the ascending activating system, and life-sustaining nuclei is more important than the secondary injury consisting of mass effect and toxic effect in the massive and basotegmental type than in the unilateral tegmental type. Similar to our study, Chung and Park also found that the prognoses of these types were not significantly different except for the unilateral tegmental type, so they suggested classifying PPH into the unilateral type and others for prognostic purposes (2).

Our study also showed that age was an indicator predicting 30 day mortality with older patients having a higher risk of death. This may be due to more co-morbidities and postoperative complications in aged patients. The result is in accordance with that found in conservatively treated investigations (10, 16). However, we did not detect age as a prognostic factor for 3-month functional outcome in multivariate regression analysis.

Besides hemorrhage volume and GCS score on admission, rostrocaudal extension mainly into the midbrain was another independent factor for 3-month functional outcome in the present study. Patients without extrapontine hemorrhage tended to gain a favorable functional outcome as no one reached a good functional recovery in the patients with rostrocaudal extension. The mechanism responsible for the difference may be attributed to the fact that surgical manipulation itself could bring about more interference to brainstem function in the longer vertical plane.

The presence of ventricular extension and hydrocephalus were regarded as risk factors in many prognostic studies, but it was not the case in the surgical patients of our study (7, 16). Ventricular extension was only significant for mortality and hydrocephalus only for functional outcome in the univariate analysis; neither was associated with outcomes 
in the multivariate analysis. We speculate that the treatment modality plays an important role in the discrepancy. In our study, surgical hematoma evacuation of both fourth ventricle and pons within 12 hours after onset was performed, so the cerebrospinal fluid pathway was opened again in early phase. Furthermore, external ventricular drainage was first done during the operation when hydrocephalus coexisted. On the contrary, a vast majority of previous studies only involved patients treated medically $(7,16)$.

Other factors such as sex, the history of hypertension, admission time, surgical modality and surgical approach which may have an impact on surgical outcome were also considered in our study, but presented no association with the surgical results.

There were several limitations in the study. Our study was a retrospective analysis with obvious inherent disadvantages. Secondly, our subjects were highly selected with GCS 4-7 on admission and hematoma volume $>5 \mathrm{ml}$, the results could not be applied to all PPH. Also, the sample size in this series was relatively small.

\section{CONCLUSION}

The treatment of patients with $\mathrm{PPH}$ continues to be a challenge to neurosurgeons. Our findings demonstrated a mortality rate of $31.1 \%$ at 1 month and a $15.6 \%$ rate of favorable functional outcome at 3 months after operation. Hematoma volume and initial GCS score, age, hemorrhagic type and rostrocaudal extension are prognostic factors for surgical outcomes. For the patients with $\mathrm{PPH}>5 \mathrm{ml}$, GCS score $<8$, those with hematoma volume less than $10 \mathrm{ml}$, GCS score $>6$, age $<65$ years, unilateral tegmental type, and absence of extrapontine hemorrhage may benefit most from surgery.

\section{ACKNOWLEDGEMENTS}

The work is funded by the National Key Technology R\&D Program for the $12^{\text {th }}$ Five-year Plan of P.R.China. The authors report no declarations of interest and are responsible for the content and writing of the paper alone.

\section{REFERENCES}

1. Balci K, Asil T, Kerimoglu M, Celik Y, Utku U: Clinical and neuroradiological predictors of mortality in patients with primary pontine hemorrhage. Clin Neurol Neurosurg 108:36-39, 2005

2. Chung CS, Park $\mathrm{CH}$ : Primary pontine hemorrhage: $A$ new $C T$ classification. Neurology 42:830-834, 1992
3. Dziewas R, Kremer M, Ludemann P, Nabavi DG, Dräger $B$, Ringelstein B: The prognostic impact of clinical and CT parameters in patients with pontine hemorrhage. Cerebrovasc Dis 16:224-229, 2003

4. Hara T, Nagata K, Kawamoto S, Sashida J, Abe T, Wada A, Sakamoto T: Functional outcome of primary pontine hemorrhage: Conservative treatment or stereotaxic surgery. No Shinkei Geka 29:823-829, 2001 (in Japanese)

5. Hong JT, Choi SJ, Kye DK, Park CK, Lee SW, Kang JK: Surgical outcome of hypertensive pontine hemorrhages: experience of 13 cases. J Korean Neurosurg Soc 27:59-65, 1998

6. Huang KB, Ji Z, Wu YM, Wang SN, Lin ZZ, Pan SY: The prediction of 30-day mortality in patients with primary pontine hemorrhage: A scoring system comparison. Eur J Neurol 19:1245-1250, 2012

7. Jang JH, Song YG, Kim YZ: Predictors of 30-day mortality and 90-day functional recovery after primary pontine hemorrhage. $J$ Korean Med Sci 26:100-107, 2011

8. Kothari RU, Brott T, Broderick JP, Barsan WG, Sauerbeck $\mathrm{LR}$, Zuccarello M, Khoury J: The ABCs of measuring intracerebral hemorrhage volumes. Stroke 27:1304-1305, 1996

9. Masiyama S, Niizuma H, Suzuki J: Pontine haemorrhage: A clinical analysis of 26 cases. J Neurol Neurosurg Psychiatry 48:658-662, 1985

10. Murata Y, Yamaguchi S, Kajikawa H, Yamamura K, Sumioka S, Nakamura S: Relationship between the clinical manifestations, computed tomographic findings and the outcome in 80 patients with primary pontine hemorrhage. J Neurol Sci 167:107-111, 1999

11. Nakajiama K: Clinicopathological study of pontine hemorrhage. Stroke 14:485-493, 1983

12. Shitamichi M, Nakamura J, Sasaki T, Suematsu K, Tokuda S: Computed tomography guided stereotactic aspiration of pontine hemorrhages. Stereotact Funct Neurosurg 54-55: 453-456, 1990

13. Rabinstein AA, Tisch SH, McClelland RL, Wijdicks EFM: Cause is the main predictor of outcome in patients with pontine hemorrhage. Cerebrovasc Dis 17:66-71, 2004

14. Takahama H, Morii K, Sato M, Sekiguchi K, Sato S: Stereotactic aspiration in hypertensive pontine hemorrhage: Comparative study with conservative therapy. No Shinkei Geka 17:733-739, 1989 (in Japanese)

15. Takeuchi S, Suzuki G, Takasato $Y$, Masaoka H, Hayakawa T, Otani $\mathrm{N}$, Yatsushige H, Shigeta K, Momose T, Wada K, Nawashiro H: Prognostic factors in patients with primary brainstem hemorrhage. Clin Neurol Neurosurg 115:732-735, 2013

16. Wessels T, Möller-Hartmann W, Noth J, Klötzsch C: CT findings and clinical features as markers for patient outcome in primary pontine hemorrhage. Am J Neuroradiol 25:257-260, 2004 